\title{
"Peliosis Hepatis" Como Complicación del Uso de Anticonceptivos Orales en una Paciente con Mielodisplasia
}

\author{
MARCELA MUÑOZ M. ${ }^{1}$, NATALIE RODRÍGUEZ Z. ${ }^{2,3}$, JUAN TORDECILLA C., ${ }^{2,3}$, \\ ESTER URETA H. ${ }^{4}$, CARLOS RIZZARDINI L. ${ }^{2}$, VERÓNICA SOTO A. ${ }^{2,5}$, KARIN WENSIOE R. ${ }^{6}$
}

1. Médico, Servicio de Pediatría. Hospital Temuco.

2. Médico, Unidad Hemato-Oncología. Hospital de Niños Roberto del Río.

3. Médico, Departamento de Pediatría, Campus Norte, Facultad de Medicina. Universidad de Chile.

4. Médico, Unidad de Imaginología. Hospital de Niños Roberto del Río.

5. Médico, Banco de Sangre. Hospital de Niños Roberto del Río.

6. Médico, Unidad de Ginecología Infantil. Hospital de Niños Roberto del Río.

\section{ABSTRACT \\ "Peliosis Hepatis" as a Complication of the Ise of Oral Contraceptives in a Patient with Myelodysplasia}

Introduction: Peliosis hepatis $(\mathrm{PH})$ is an uncommon condition in pediatrics; however, it is one of the most serious complications associated with the long-term use of use of steroids. It is characterized by multiple blood-filled cavities, mostly involving the liver. Myelodysplastic Syndrome (MDS) is also a complex and infrequent hematological condition; it may transform into acute leukemia and its treatment requires medications that may lead to PH. Case Report: 13 year-old girl with MDS, refractory cytopenia type. A family donor for SCT was not available, therefore immunosuppressive treatment, steroids and transfusions were initiated. Due to metrorrhage, estrogen was used at high doses. She developed acute abdominal pain; abdominal ultrasound and CT scan showed PH and peritoneal bleeding. Oral contraceptives were decreased resulting in reduction of $\mathrm{PH}$, but a new episode of uterine bleeding causing hypovolemic shock forced a hysterectomy in order to suspend estrogen treatment. Due to lack of response to treatment to SMD, she continued been treated with transfusions as needed, and died 32 months post diagnosis. Discussion: $\mathrm{PH}$ is an uncommon and life-threatening condition in children receiving prolonged treatment with steroids. Current modalities of SCT in patients with MDS will replace the need for steroids, thus avoiding this severe complication.

(Key words: Myelodysplastic Syndrome, Peliosis hepatis, steroids, stem cell transplantation).

Rev Chil Pediatr 2009; 80 (4): 354-360

Trabajo recibido el 17 de julio de 2008, devuelto para corregir el 03 de octubre de 2008, segunda versión el 12 de enero de 2009, aceptado para publicación el 06 de abril de 2009.

Correspondencia a:

Dra. Marcela Muñoz M.

E-mail:mmunozmillan@gmail.com 


\section{RESUMEN}

Introducción: La Peliosis Hepática (PH) es una condición muy infrecuente en pediatría, caracterizada por la presencia de múltiples cavidades sanguíneas en el parénquima hepático, asociada al uso prolongado de estrógenos o corticoides, El Síndrome Mielodisplásico (SMD) es una alteración hematológica compleja que puede evolucionar a leucemia y que puede requerir para su tratamiento medicamentos relacionados al desarrollo de PH. Caso Clínico: Niña 13 años, con SMD tipo citopenia refractaria, con dependencia transfusional, sin posibilidad de realizar Trasplante de progenitores hematopoyéticos (TPH) por falta de donante familiar compatible. Recibió transfusiones, inmunosupresores y corticoides por tiempo prolongado. Presentó metrorragias severas requiriendo estrógenos en altas dosis. Evolucionó con hemoperitoneo, diagnosticándose $\mathrm{PH}$ por ecografía y scanner abdominal. Al reducir dosis de estrógenos disminuyeron lesiones hepáticas, pero nuevo episodio de metrorragia con shock hipovolémico, obligó a realizar histerectomía para suspender estrógenos. Sin respuesta a tratamiento del SMD, se mantuvo con transfusiones según requerimiento y falleció a los 32 meses del diagnóstico. Discusión: La PH es una complicación grave, que podría evitarse con el desarrollo de nuevas técnicas de TPH que permiten contar con donantes no relacionados para el tratamiento de síndromes de falla medular como el SMD.

(Palabras clave: Síndrome mielodisplásico, peliosis hepática, esteroides, trasplante de progenitores hematopoyéticos).

Rev Chil Pediatr 2009; 80 (4): 354-360

\section{Introducción}

La "peliosis hepatis" o peliosis hepática $(\mathrm{PH})$, es un trastorno benigno de baja frecuencia, descrito por Wagner en 1861 y por Schoenlak en 1916, quien introdujo el término "peliosis", que significa "púrpura", debido al color observado en el tejido hepático por extravasación sanguínea. Se caracteriza por la presencia de cavidades quísticas con contenido sanguíneo, de forma, tamaño y distribución variable en el hígado. También ha sido descrita en pulmón y otros órganos que pertenecen al sistema retículo endotelial como bazo y ganglios linfáticos ${ }^{1,2}$.

En adultos la $\mathrm{PH}$ se ha relacionado con condiciones patológicas como tuberculosis, fibrosis quística e infección por VIH. También ha sido notificada después del uso de fármacos como esteroides anabólicos, azatriopina, 6tioguanina, 6-mercaptopurina y anticonceptivos. $\mathrm{La} \mathrm{PH}$ en niños, ha sido asociada a condiciones crónicas como fibrosis quística, malnutrición, Anemia de Fanconi, tumores adrenales, Síndrome de Marfan, cardiopatías congénitas o posterior a trasplante renal ${ }^{1-5}$.

El Síndrome Mielodisplásico (SMD) es una alteración hematológica poco frecuente en pediatría que puede evolucionar a falla medular y leucemia, requiriendo tratamientos prolongados y complejos que pueden asociarse a complicaciones como la $\mathrm{PH}$.

La sospecha precoz de este tipo de complicación, que puede ser de riesgo vital para el paciente, permite un manejo oportuno y efectivo, disminuyendo la morbimortalidad asociada. Los avances en las técnicas de Trasplante de progenitores hematopoyéticos (TPH) en relación al tratamiento del SMD y de otros síndromes de falla medular, han permitido disminuir los riesgos asociados al uso prolongado de esteroides.

Con el objetivo de sensibilizar a los pediatras y hematólogos pediatras sobre la importancia de una sospecha dirigida y precoz de una complicación poco frecuente, reportamos un caso de $\mathrm{PH}$ en una paciente adolescente con diagnóstico de SMD tipo citopenia refractaria, en tratamiento con anticonceptivos orales y esteroides por un largo período.

\section{Caso Clínico}

Paciente de 13 años, sexo femenino, con diagnóstico de SMD desde agosto 2002 por pancitopenia: Hematocrito (Hto) 17\%, Hemoglobina $(\mathrm{Hb}) 5,8 \mathrm{~g} / \mathrm{dL}$, Volumen Corpuscular Me- 
dio (VCM) $102 \mathrm{fL}$, reticulocitos 1,6\%, leucocitos $3200 \times \mathrm{mm}^{3}$, recuento absoluto de neutrófilos (RAN) 1 536, y plaquetas $17000 \mathrm{x} \mathrm{mm}^{3}$. Mielograma con celularidad aumentada, presencia de megacariocitos, displasia eritroide, con precursores multinucleados, disociación de la maduración núcleo-citoplasma y eritroblastos con restos nucleares en el citoplasma. La serie granulocítica mostró hipogranulación, alteración en la distribución de los gránulos y pseudopelger (figura 1). Además hemoglobina fetal 2,9\% (valor normal hasta $2 \%$ ) y estudio citogenético de médula ósea normal, por lo que se diagnosticó SMD tipo citopenia refractaria.

La paciente inició tratamiento según Protocolo del Programa Infantil de Drogas Antineoplásicas (PINDA) con ácido fólico, multivitamínicos, vitamina $\mathrm{C}$, ácido tranexámico y transfusiones de glóbulos rojos y plaquetas según requerimientos.
Evolucionó durante el año 2002 con episodios repetidos de metrorragia que requirieron hospitalizaciones frecuentes, uso de anovulatorios a permanencia (dosis de estrógeno $30 \mathrm{ug}$ día), ácido tranexámico y transfusión de glóbulos rojos y plaquetas.

Debido a su evolución, con dependencia de transfusiones cada 21 días, se planteó como principal alternativa terapéutica un TPH, procedimiento que no pudo realizarse por no disponer de un donante familiar compatible, que era la indicación según protocolo en ese momento.

Debido a metrorragias repetidas se aumentó la dosis de estrógenos hasta 90 ug por día para lograr atrofia del endometrio. Se realiza biopsia de médula ósea que confirma hipoplasia severa, con ausencia de blastos (figura 2). Debido a la evolución con hipoplasia medular, se efectúa tratamiento inmunosupresor con linfoglobulina antitimocítica de origen equino en do-
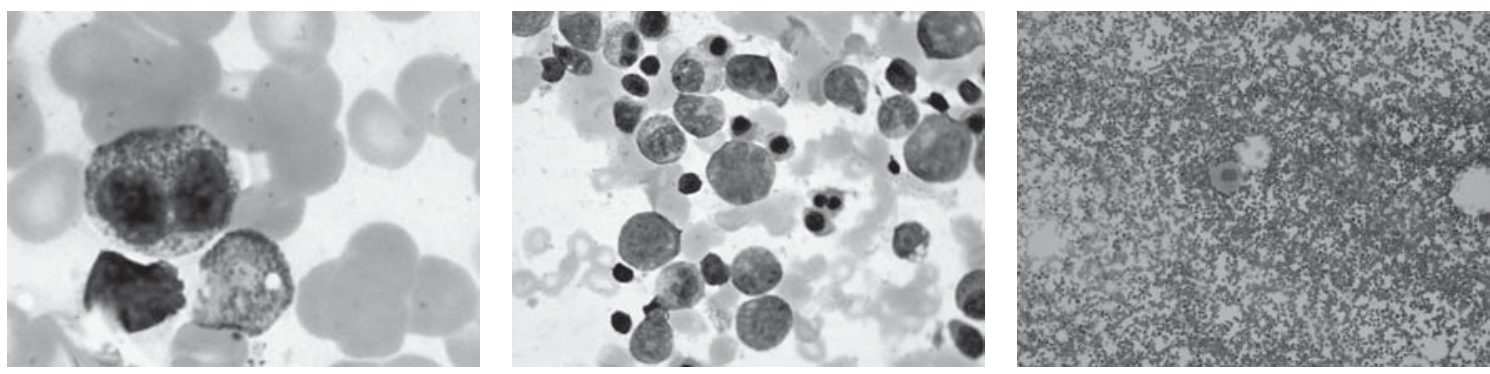

Figura 1. Mielograma al diagnóstico. Hipercelularidad y signos de displasia en serie roja (eritroblastos multinucleados) y blanca (alteración distribución gránulos y pseudopelger).
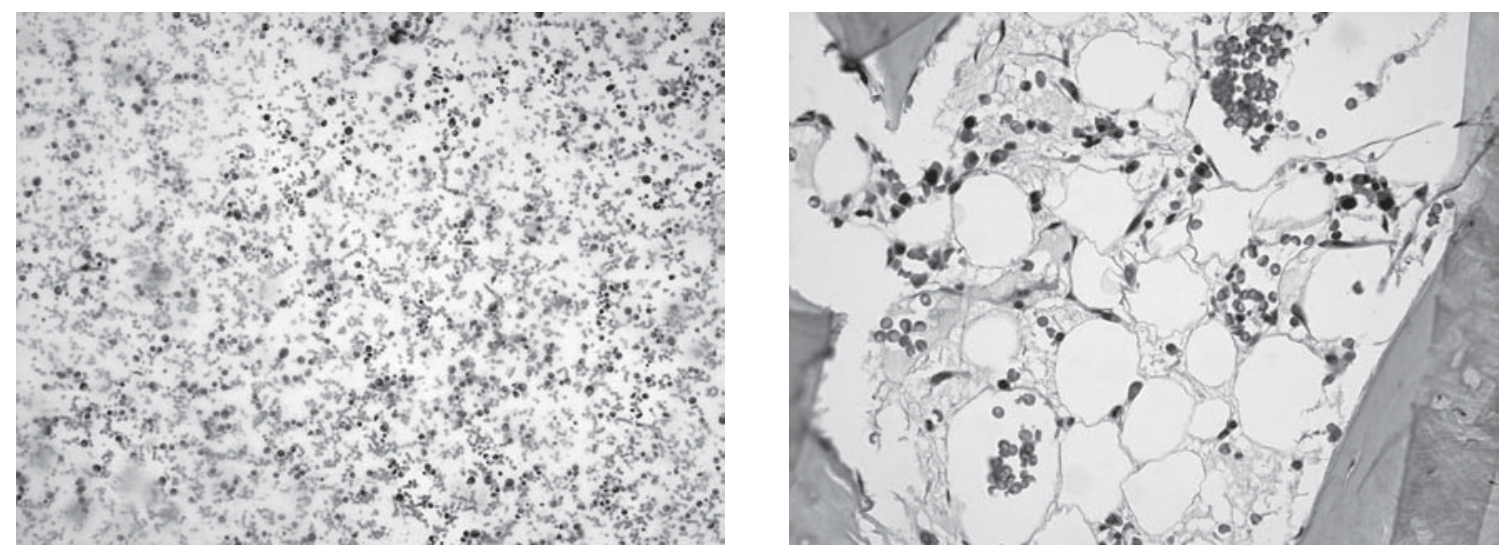

Figura 2. Mielograma y biopsia de médula ósea: hipoplasia medular severa sin blastos. 


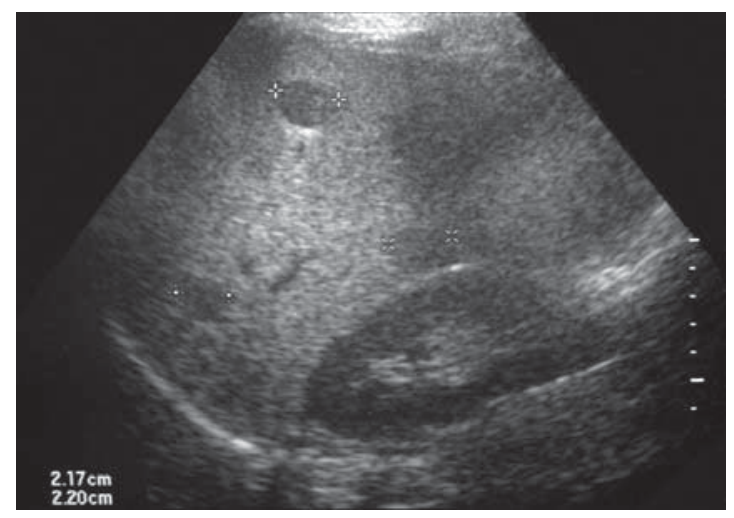

Figura 3. Ecotomografía abdominal. Múltiples imágenes hipoecogénicas, redondeadas, bien delimitadas, no vascularizadas en evaluación con doppler color, que se distribuyen en ambos lóbulos hepáticos, existiendo dos de mayor tamaño, de 7 y $5 \mathrm{~cm}$ localizadas en los segmentos IV y V.

sis de $15 \mathrm{mg} / \mathrm{kg} /$ día por cinco días; ciclosporina en dosis de $10 \mathrm{mg} / \mathrm{kg} /$ día y metilprednisolona en dosis de $5 \mathrm{mg} / \mathrm{kg} /$ día durante cinco días, luego prednisona oral en dosis de $1 \mathrm{mg} / \mathrm{kg} /$ día hasta el día 28. A partir de ese día, la dosis de corticoides se mantuvo en 7,5 mg/día. Presenta respuesta parcial a tratamiento inmunosupresor a los 90 días, persistiendo hipocelularidad y ausencia de megacariocitos en el mielograma de control.

A los 2 años de evolución, consulta por dolor abdominal difuso, tipo cólico, de inicio agudo. El hemograma mostró leucocitos $3600 \mathrm{x} \mathrm{mm}^{3}$, Hto $17 \%$, Hb $5 \mathrm{~g} / \mathrm{dL}$ y plaquetas de $5000 \mathrm{x}$ $\mathrm{mm}^{3}$. En la ecografía y tomografía computada abdominal destaca hígado con múltiples imágenes hipoecogénicas distribuidas en ambos lóbulos hepáticos, de 5 y $7 \mathrm{~cm}$ de diámetro ubicadas en los segmentos hepáticos IV y V respectivamente (figuras 3 y 4), sugerentes de $\mathrm{PH}$. El diagnóstico se plantea por las imágenes radiológicas y por las condiciones de la paciente no se realiza biopsia hepática.

Se decide suspender estrógenos y para disminuir posibilidad de metrorragia masiva con riesgo vital, se plantea histerectomía. Se presenta su caso clínico en comité de ética de nuestro hospital y, de acuerdo con la paciente y su familia, se planifica una histerectomía. Previo a esta intervención presenta episodio de hemoperitoneo, el que fue manejado médicamente. Posterior a su estabilización clínica, se

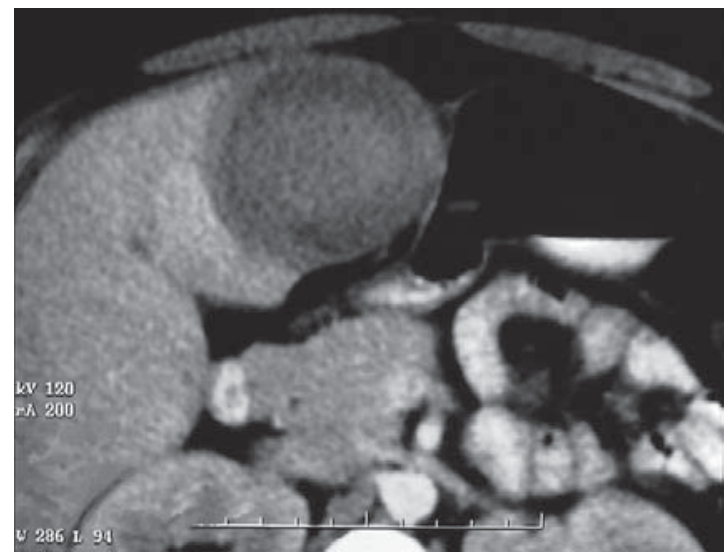

Figura 4. Tomografía computada abdominal. Contenido espontáneamente hiperdenso en las lesiones hepáticas de mayor tamaño, sugerente de sangrado reciente intralesional.

realizó histerectomía sin complicaciones. Un mes después de la cirugía se repitió infusión de linfoglobulina, corticoides y ciclosporina, sin respuesta hematológica. Luego de la suspensión de estrógenos, se observó en los controles ecográficos regresión del tamaño de las lesiones hepáticas, sin resolución completa.

Por falta de respuesta del SMD al tratamiento médico realizado, se inicia cuidado paliativo, trasladándose a su domicilio en el norte del país, donde continuó en contacto, vía telefónica, con el equipo médico de nuestra unidad y se mantuvo con transfusiones de glóbulos rojos y plaquetas según condición clínica en el hospital de su ciudad. Fallece por probable hemorragia intracraneana a los 2 años 8 meses del diagnóstico. No se realizó autopsia.

\section{Discusión}

Creemos interesante presentar el caso de una paciente adolescente con SMD que evolucionó con una $\mathrm{PH}$, complicación poco frecuente asociada a su tratamiento y realizar una revisión actualizada de la literatura en relación a esta condición clínica.

El SMD constituye un grupo heterogéneo de alteraciones clonales de la célula madre hematopoyética totipotencial, caracterizado por hematopoyesis inefectiva asociado con médula 
ósea frecuentemente hipercelular y citopenias periféricas de severidad variable. Su frecuencia es de 0,5 a 1,5 casos por millón en niños menores de 15 años. Su incidencia es relativamente baja, lo que corresponde a un 3 a $5 \%$ de las enfermedades hematológicas malignas de la infancia, pero representa un grupo de pacientes de difícil manejo que constituyen un desafío para el hemato-oncólogo. La evolución natural del SMD puede caracterizarse por una progresión a pancitopenia, ocasionando la muerte por hemorragia severa y/o infecciones graves. Por otro lado, un 20 a $30 \%$ de los casos evolucionan a leucemia aguda de tipo mieloide en el plazo de un año aproximadamente. El tratamiento actual de la citopenia refractaria con dependencia transfusional o con exceso de blastos debe ser el TPH, lo que aumenta significativamente las probabilidades de sobrevida $^{5,6}$.

El SMD se puede clasificar en: SMD asociado a enfermedad genética o alteraciones cromosómicas, como Síndrome de Down o Neurofibromatosis; SMD secundarios a quimioterapia previa y SMD primarios, como citopenia refractaria, citopenia refractaria con exceso de blastos, citopenia refractaria con exceso de blastos en transformación y leucemia mielomonocítica juvenil ${ }^{6,7}$.

El caso que presentamos es de una paciente con SMD tipo citopenia refractaria, que debutó con una médula ósea hipercelular, pero que evolucionó hacia una hipoplasia medular severa. Este SMD hipoplásico tendría un mecanismo fisiopatológico similar al descrito en los casos de Anemia aplástica. La evidencia actual demuestra que la inhibición de células progenitoras mediada por linfocitos $\mathrm{T}$, puede causar pancitopenia en ambos trastornos, y las células progenitoras hematopoyéticas pueden ser anormalmente susceptibles a la acción de linfocitos T, debido a la sobreexpresión del receptor Fas, lo que favorece la apoptosis de estas células a nivel medular. En este tipo de pacientes si no es posible realizar TPH, se plantea como alternativa la inmunomodulación con linfoglobulina antitimocito y ciclosporina. En la literatura se describe que aquellos pacientes que presentan médula ósea hipoplásica, cariotipo normal $\mathrm{y}<5 \%$ de blastos tienen una posibilidad de respuesta a este tipo de tratamiento que varía de 34 a 47\%. Nuestra paciente no respondió completamente a la terapia inmunosupresora, sin embargo, se consiguió disminuir la frecuencia de transfusiones de glóbulos rojos y plaquetas durante un tiempo ${ }^{8-11}$.

La evolución hacia una pancitopenia persistente con una médula hipoplásica, se manifestó principalmente con trastornos hemorrágicos, que complicaron gravemente la evolución de la paciente, siendo algunas de las hemorragias de riesgo vital. Con el objetivo de poder controlar estos síntomas y no disponiendo de un donante familiar para TPH de acuerdo a las indicaciones de ese momento, se usó por tiempo prolongado corticoides, ciclosporina y estrógenos, siendo esta asociación de fármacos el factor gatillante de la $\mathrm{PH}$, como se describe en la literatura.

La PH es una condición benigna muy infrecuente, que se caracteriza por la presencia de múltiples cavidades de forma irregular y tamaño variable, con contenido sanguíneo, que se distribuyen principalmente en el parénquima hepático. La causa es desconocida, pero se han descrito distintas asociaciones con enfermedades malignas, infecciones como VIH y tuberculosis, diabetes mellitus, falla renal y fármacos, como andrógenos anabólicos y anticonceptivos orales. Sin embargo, en un $25-50 \%$ de los casos no hay una condición identificada. Se han postulado numerosas teorías para explicar la fisiopatología de la PH. Estas incluyen la obstrucción del flujo sanguíneo centrolobular y sinusoidal, necrosis hepatocelular y efectos tóxicos en la barrera sinusoidal ${ }^{1-5,12,13}$.

La presentación clínica es variable, pudiendo ser asintomática, con lesiones focales hepáticas diagnosticadas como hallazgo en un examen radiológico, puede debutar como hepatomegalia o disfunción hepática y con menor frecuencia manifestarse inicialmente con complicaciones tales como falla hepática, colestasis, hipertensión portal y hemoperitoneo secundario a ruptura espontánea de una lesión hepática. La evolución natural de la $\mathrm{PH}$ es desconocida, puede fluctuar desde la resolución de las lesiones a falla hepática o hemorragia intraperitoneal fatal ${ }^{1-5}$.

La tomografía computada (TC) con con- 
traste permite una mejor caracterización de las lesiones. Se observan zonas de aspecto heterogéneo e hipodensidad con respecto al parénquima no comprometido. En la fase arterial hay zonas no perfundidas con dilatación vascular sin evidencias de shunts y también lesiones de gran tamaño que presentan un centro que gana contraste y que en la fase portal presentan progresión centrífuga, pudiendo encontrarse acumulación de contraste en estas áreas en fase tardía, llegando a observarse isodensas en la medida que transcurre la evaluación. Estas lesiones no ejercen efecto de masa, lo que las diferencia de lesiones de tipo metastático o tumoral. También pueden verse hematomas de localización subcapsular, en distintos estados de evolución y en aquellos casos en que existe sangramiento activo al momento del examen se puede ver extravasación del contraste ${ }^{14-18}$.

El diagnóstico diferencial debe hacerse principalmente con adenomas, que se comportan como hiperdensos en fase arterial y se hacen isodensos con el resto del parénquima en fase portal. También se debe diferenciar del hemangioma, que presentará llene desde la periferia hacia el centro en la fase arterial, para hacerse isodenso en fase portal. En el caso de las hiperplasias nodulares, éstas son hipervasculares en fase arterial, haciéndose isodensas en fases portal y tardía. Por último las metástasis hipervasculares en fase arterial se hacen hipodensas en fases tardías debido al lavado rápido de contraste y por otro lado frecuentemente producen distorsión de la trama vascular ${ }^{15,19}$.

La Resonancia Magnética puede mostrar un patrón normal o lesiones levemente hiperintensas en secuencias $\mathrm{T} 1$ y T2, pudiendo agregarse pequeños focos hiperintensos debido a la presencia de metahemoglobina $a^{4,14,20,21}$.

La PH se debe sospechar en pacientes con tratamiento esteroidal prolongado y/o uso de anticonceptivos orales, dolor abdominal e imágenes sugerentes. Su tratamiento debe ser multidisciplinario y debe incluir apoyo intensivo de medicina transfusional. En la actualidad, el desarrollo de alternativas terapéuticas más efectivas en el manejo de los Síndromes de falla medular (Anemia de Fanconi, Anemia de Blackfan Diamond, entre otros) y de los SMD, incluido el TPH de donante no relacionado en algunos casos seleccionados, podrían evitar complicaciones graves asociadas al tratamiento prolongado con esteroides y otros fármacos, mejorando las posibilidades de sobrevida en este grupo de pacientes de manejo muy complejo.

\section{Referencias}

1.- Samyn M, Hadzic N, Davenport A, et al: Peliosis Hepatis in Childhood: Case Report and Review of the Literature. J Pediatr Gastroenterol Nutr 2004; 39: 4314.

2.- Jacquemin E, Pariente D, Fabre M, et al: Peliosis hepatis with initial presentation as acute hepatic failure and intraperitoneal hemorrhage in children. J Hepatol 1999; 30: 1146-50.

3.- Joseph F, Younis N, Haydon G, et al: Peliosis of the spleen with massive recurrent haemorrhagic ascites, despite splenectomy, and associated with elevated levels of vascular endothelial growth factor. Eur J Gastroenterol Hepatol 2004; 16: 1401-6.

4.- Hiorns $M$, Rossi U, Roebuck D: Peliosis Hepatis causing inferior vena cava compression in a 3 year old child. Pediatr Radiol 2005; 35(2): 209-11.

5.- Resnick I, Salvin S, Or R, et al: Peliosis Hepatis following treatment with androgen-steroids in patients with bone marrow failure síndromes. Hematologica 2007; 92: 106-10.

6.- Tordecilla J, Bravo M, Campbell M, et al: Síndrome mielodisplásico en pediatría: experiencia clínica. Rev Chil Pediatr 1999; 70 (5): 376-83.

7.- Kurzrock R: Myelodysplastic Síndrome Overview. Seminars in Hematology 2002; 39 (3): 18-25.

8.- Barret J, Saunthararajah Y, Molldrem J: Myelodysplastic Syndrome and Aplastic Anemia: Distinct entities or diseases linked by a common pathophysiology. Seminars in Hematology 2001; 37 (1): 15-29.

9.- Yazji S, Giles F, Tsimberidou A, et al: Antithymocyte globulin (ATG) based therapy in patients with myelodisplastic syndromes. LeuKemia 2003; 17: 21016.

10.- Killick S, Mutfi G, Cavenagh J, et al: A pilot study of antithymocyte globulin (ATG) in the treatment of patients with "Low risk" myelodysplasia. Br Jour of Haematol 2003; 120: 679-84.

11.- Orimoto M, Maeda M, Cai L, et al: Successful immunosuppresive therapy for a child with myelodysplastic síndrome. Med Ped Oncol 2003; 40 (5): 334-5.

12.- Tsokos M, Püschel K: Isolated peliosis of the spleen. 
Report of 2 autopsy cases. Am J Forensic Med Pathol 2004; 25: 251-4.

13.- Tsokos M, Erbersdobler A: Pathology of peliosis. Forensic Sci Int 2005; 149 (1): 25-33.

14.- Rumack $C$, Wilson $S$, Charboneau $W$ : Diagnóstico por Ecografía. Ed. Elsiever 2006: 2 (4): 111-2.

15.- Kleinig P, Davies R, Maddern G: Peliosis Hepatis: Central "Fast Surge" Ultrasound Enhancement and Multislice CT Appearances. Clin Radiol 2003; 58: 9958.

16.- Gouya H, Vignaux O, Legmann P: Peliosis hepatic: triphasic helical CT and dynamic MRI findings. Abdom Imaging 2001; 26: 507-9.

17.- Vignaux $O$, Legmann $P$, de Pinieux $G$, et al: Hemorraghic necrosis due to peliosis hepatis: imaging findings and pathological correlation. Eur Radiol 1999; 9: 4546 .

18.- Ferrozzi F, Tognini G, Zuccoli G: Peliosis hepatic with pseudotumoral and hemorrhagic evolution: CT and MR findings. Abdom Imaging 2001; 26: 197 9.

19.- Jamadar D, D'Souza S, Thomas E: Case Report: Radiological appearances in peliosis hepatic. Br J Radiol 1994; 67: 102-4.

20.- Dal M, Dong H, Chul H: Case Report: Imaging findings of hepatic Sinusoidal Dilatation. AJR 2004; 183: 1075 7.

21.- Wang S, Ruggles S, Vade A: Hepatic Rupture Caused by Peliosis Hepatis. J Pediatr Surg 2001; 36 (9): 14569 